\title{
SPIN 3/2 INTERACTING FIELDS AND HEAVY BARYON CHIRAL PERTURBATION THEORY
}

\author{
M. Napsuciale and J.L. Lucio M. \\ Instituto de Física, Univiversidad de Guanajuato \\ Apartado Postal E-143, León, Gto., México
}

\begin{abstract}
.
We analyze the consistency of the Chiral Lagrangian approach to the description of the spin $3 / 2$ interacting theory. We argue that to lowest order in the $1 / \mathrm{m}$ expansion, the formalism leads to the appropriated constraints and the theory is free of the so called "off shell" ambiguities.
\end{abstract}

\footnotetext{
${ }^{1}$ Work supported by CONACyT under contract 4918-E
} 
Chiral Perturbation Theory is a useful tool in the description of low momentum process involving Goldstone Bosons [1]. When the formalism is extended to include interactions with Baryons it suffers from two unpleasant characteristics i) There is no correspondence between the loop and momentum expansion. ii) The expansion parameter turn out to be of order one. A new formalism to circumvent the problems arising in Baryon Chiral Perturbation Theory (BChPT) was introduced by Jenkins and Manohar (J-M) [2]. The approach of $\mathrm{J}-\mathrm{M}$ is based on the technics originally developed to describe heavy quark systems [3], which amounts to consider the baryons as heavy static sources of Golstone Bosons. The effective theory is written in terms of definite velocity fields for which the Dirac equation correspond to a massless baryon. The heavy baryon Lagrangian has a $1 / \mathrm{m}$ expansion, the $1 / \mathrm{m}$ effects in the original theory are reproduced in the effective theory by including higher dimension operators suppressed by inverse powers of $\mathrm{m}$.

Besides the baryon octet the decuplet is also included in the effective theory. In this case the theory involves spin $3 / 2$ fields whose effective mass is $\Delta m=M-m$ (m-M masses of the baryon octet and decuplet respectively). It is possible to construct an effective theory with only octet baryons and Goldstone Bosons by integrating out the decuplet fields. Decuplet effects in the original theory are reproduced in the new theory by higher dimension operators involving only octet baryons and mesons. These higher dimension operators are suppressed by inverse powers of $\Delta m$. In the real world the decuplet-octet baryon mass difference $\Delta m \approx 300 \mathrm{MeV}$ which is small compared to the hadronic scale of $1 \mathrm{GeV}$. The smallness of $\Delta m$ produces an enhancement of the decuplet effects and for that reason it is best to retain explicit decuplet fields in the effective theory, rather than integrate them out. This formalism has been applied to study the $T \rightarrow B P$ and $T \rightarrow B \gamma$ decays [4] (T,B,P stand for decuplet, baryon and meson octets respectively). The next to leading order of Baryon Chiral Perturbation Theory with $S=1 / 2$ baryons and Goldstone bosons have also been examined [5].

It is well known that Quantum Field Theory for spin 3/2 interacting fields suffers of serious incosistencies. In particular, within the Rarita-Schwinger formalism [6] the R-S spinor $\psi_{\mu}$ has more degrees of freedom than required, which makes the classical Lagrangian non-unique. In fact there exist a whole family of one parameter Lagrangians $\mathcal{L}(A)$ from which the Dirac-Fierz-Pauli 
equation and the necessary free field constraints for $\psi_{\mu}$ are obtained [7]. Furthermore, these Lagrangians remain invariant under point transformations (R-invariance) which mix the spurious spin $1 / 2$ fields contained in $\psi_{\mu}$. This invariance ensures that the unphysical spin $1 / 2$ degrees of freedom have no observable effects. Generalizations of this scheme to describe interacting spin $3 / 2$ fields requires that the corresponding Lagrangian preserves $\mathrm{R}$ invariance, this fact introduces new ambiguities into the theory (the so-called "off-shell" parameters) [8]. It should also be emphasized that the constraints equations, which ensure the elimination of the unphysical spin $1 / 2$ degrees of freedom in the free case, are spoiled by the interaction.

It is our purpose in this paper to analyze the consistency of the Chiral Lagrangian approach to the description of the spin $3 / 2$ interacting theory. We construct an $\mathrm{R}$ invariant Lagrangian and argue that only to lowest order in the $1 / \mathrm{m}$ expansion, the approach leads to the appropriated constraints and the theory is free of the so called "off-shell" ambiguities.

\section{FREE FIELD THEORY}

Within the Rarita-Schwinger approach, the $\mathrm{S}=3 / 2$ field is described by a spinor-vector $\psi_{\mu}$ which is obtained from the tensor product of a spinor and a four-vector. Clearly $\psi_{\mu}$ involves more degrees of freedom than required to describe a spin $3 / 2$ field. This redundancy is reflected in the formalism in the need of the subsidiary conditions:

$$
\begin{aligned}
& \gamma^{\mu} \psi_{\mu}(x)=0 \\
& \partial^{\mu} \psi_{\mu}(x)=0
\end{aligned}
$$

It has been shown that for the spin $3 / 2$ field, there exist a whole family of one parameter Lagrangians from wich the equation of motion and the subsidiary conditions can be derived [7]

$$
\mathcal{L}(A)=\psi^{\mu}(x)\left\{i \partial_{\alpha} \Gamma_{\mu \nu}^{\alpha}(A)-M B_{\mu \nu}(A)\right\} \psi^{\nu}(x)
$$

where 


$$
\begin{aligned}
\Gamma_{\mu \nu}^{\alpha}(A) & =g_{\mu \nu} \gamma^{\alpha}+B \gamma_{\mu} \gamma^{\alpha} \gamma_{\nu}+A\left(\gamma_{\mu} g^{\alpha}{ }_{\nu}+g_{\mu}{ }^{\alpha} \gamma_{\nu}\right) . \\
B_{\mu \nu}(A) & =g_{\mu \nu}-C \gamma_{\mu} \gamma_{\nu} \\
A \neq-1 / 2 & , \quad B=\frac{3}{2} A^{2}+A+\frac{1}{2}, \quad C=3 A^{2}+3 A+1
\end{aligned}
$$

For $\mathrm{A}=-1 / 3, \mathcal{L}(A)$ reduces to the Lagrangian originally proposed by R-S [6]. From (2) the spin $\frac{3}{2}$ field propagator is obtained

$$
i \Delta_{\mu \nu}(P, A)=\frac{\Sigma_{\mu \nu}(P, A)}{P^{2}-M^{2}+i \epsilon}
$$

with

$$
\begin{aligned}
\Sigma_{\mu \nu}(P, A)=2 M S_{\mu \nu} & -\frac{1}{6} \frac{A+1}{2 A+1} \frac{P^{2}-M^{2}}{M}\left\{\gamma_{\mu}\left(\frac{2 P}{M}-\gamma\right)_{\nu}\right. \\
& \left.+\left(\frac{2 P}{M}-\gamma\right)_{\nu} \gamma_{\mu}-\frac{A+1}{2 A+1}\left(\gamma_{\mu} \frac{P}{M} \gamma_{\nu}-2 \gamma_{\mu} \gamma_{\nu}\right)\right\}
\end{aligned}
$$

and

$$
S_{\mu \nu}=\left\{-g_{\mu \nu}+\frac{1}{3} \gamma_{\mu} \gamma_{\nu}-\frac{1}{3 M}\left(\gamma_{\mu} P_{\nu}-P_{\mu} \gamma_{\nu}\right)+\frac{2}{3 M^{2}} P_{\mu} P_{\nu}\right\} \frac{P+M}{2 M}
$$

The following properties of the Lagrangian are worth-remarking

- R Invariance. By construction the Lagrangian (2) is invariant under the point transformations:

$$
\psi_{\mu} \rightarrow \psi_{\mu}^{\prime}=R_{\mu \alpha}(a) \psi^{\alpha} \quad A \rightarrow A^{\prime}=\frac{A-2 a}{1+4 a}
$$

where

$$
R_{\mu \nu}(a)=g_{\mu \nu}+a \gamma_{\mu} \gamma_{\nu} \quad a \neq-\frac{1}{4}
$$


The $\mathrm{R}$ operator acts only on the spin $1 / 2$ content of $\psi_{\mu}$. The arbitrariness of the spin $1 / 2$ components of $\psi_{\mu}$ is at the origin of the family of one parameter Lagrangians (2).

Useful properties of the $\mathrm{R}$ operator are

$$
\begin{aligned}
R_{\mu \nu}(a) R_{\lambda}^{\nu}(b) & =R_{\mu \lambda}(a+b+4 a b) \\
R_{\mu \nu}^{-1}(a) & =R_{\mu \nu}\left(-\frac{a}{4 a+1}\right) \\
R_{\mu \nu}(0) & =g_{\mu \nu}
\end{aligned}
$$

- A Independence. The generalization of (2) to include interactions should lead to physical quantities which are A independent. This is assured by the Kamefuchi-O'Raifeartaigh-Salam [9] theorem. A proof of the theorem in this particular case is given by Urias [7] based on the observation that.

$$
\mathcal{L}(A)=\bar{\psi}^{\mu} K_{\mu \nu}(A) \psi^{\nu}
$$

where

$$
K_{\mu \nu}(A)=R_{\mu \alpha}\left(\frac{1}{2}(1+3 A)\right) \mathcal{K}^{\alpha \beta} R_{\beta \nu}\left(\frac{1}{2}(1+3 A)\right)
$$

and $\mathcal{K}^{\alpha \beta}$ is the operator corresponding to $A=-1 / 3$

$$
\mathcal{K}^{\alpha \beta}=(\not P-M) g^{\alpha \beta}+\frac{1}{3}\left(\gamma^{\alpha} \mathbb{P} \gamma^{\beta}-P^{\alpha} \gamma^{\beta}-\gamma^{\alpha} P^{\beta}+M \gamma^{\alpha} \gamma^{\beta}\right) .
$$

An application of this factorization property to describe the $\pi p$ elastic scattering in the $\Delta$ region has been reported in [10].

\section{INTERACTIONS.}

We restrict our attention to the strong interactions of spin $3 / 2$ baryons, electromagnetic interactions can be introduced by gauging the theory. There exist abundant literature $[8,12,13]$, about the inconsistencies of the first and 
second quantized version of the interacting spin 3/2 field theory. Further ambiguities associated to the so-called "off-shell" parameters (to be discussed below) have also been analyzed [13]. In spite of the difficulties previously mentioned, chiral symmetry has been traditionally taken as the guiding principle to construct phenomenological Lagrangians aiming to describe the existing data $[11,14]$. Further requirements for any sensible Lagrangians are:

R-Invariance. The full Lagrangian, $\mathcal{L}=\mathcal{L}_{\text {free }}+\mathcal{L}_{\text {int }}$ must be invariant under contact $\mathrm{R}$ transformations. This ensures, trough the K-O-S theorem [9] the A independence of physical quantities.

Constraints. Once the interactions are included, the Lagrangian must lead to the appropiated constraints so that only the correct number of degrees of freedom to describe a spin 3/2 field are left.

A convenient procedure to construct $\mathrm{R}$ invariant Lagrangian describing interacting spin $3 / 2$ fields can be envisaged paralleling the factorization property (6) of the free Lagrangian. For definitiveness consider the $\frac{3}{2}^{+} \frac{3}{2}^{+} 0^{-}$interaction. Using the nonlinear realization of chiral symmetry the Lagrangians has the Lorentz structure (In the following we omit the SU(3) structure of the interaction as it is of no relevance for our argument)

$$
\mathcal{L}_{\frac{3}{2} \frac{3}{2} 0}=\bar{\psi}^{\mu} O_{\mu \nu \alpha} \psi^{\nu} \Delta^{\alpha}
$$

Where $\psi_{\mu}, \psi$ stand for the decuplet tensor and octet baryon matrix respectively and $\Delta^{\nu}$ stand for the axial vector field

$$
\Delta^{\mu}=\frac{i}{2}\left(\xi \partial^{\mu} \xi^{+}-\xi^{+} \partial^{\mu} \xi\right)
$$

with $\xi=\exp \left(\frac{i}{f_{\pi}} \pi\right), \pi$ being the pseudoscalar field matrix.

The simplest possibility for $O_{\mu \nu \alpha}$ is

$$
O_{\mu \nu \alpha}^{S}=g_{\mu \nu} \gamma_{\alpha} \gamma_{5}
$$

Since all the Lagrangian related by an $\mathrm{R}$ transformation are equivalent [7], a family of A dependent Lagrangians is obtained by applying the point transformations (5) 


$$
O_{\mu \nu \alpha}(A)=R_{\mu \beta}(h(A)) g^{\beta}{ }_{\sigma} \gamma_{\alpha} \gamma_{5} R_{\nu}^{\sigma}(\ell(A))
$$

$h(A), \ell(A)$ are, in principle, arbitrary functions of $\mathrm{A}$, however the requirement of $\mathrm{R}$ invariance of the Lagrangian limits $h(A)$ and $\ell(A)$ to the class of functions for which the following identity holds:

$$
R_{\mu}{ }^{\delta}(a) O_{\delta \beta \alpha}\left(A^{\prime}\right) R^{\beta}{ }_{\nu}(a)=O_{\mu \nu \alpha}(A)
$$

Using the composition properties (5) of the R operators, (8) reduces to the pair of equations

$$
a+(1+4 a) h\left(A^{\prime}\right)=h(A) ; \quad a+(1+4 a) \ell\left(A^{\prime}\right)=\ell(A)
$$

For linear functions of $\mathrm{A}$, the solution for $\ell(A), h(A)$ are (X,Y arbitrary parameters);

$$
\ell(A)=\frac{1}{2}(1+4 X) A+X ; \quad h(A)=\frac{1}{2}(1+4 Y) A+Y
$$

A similar analysis can be applied to the $\frac{3}{2}^{+} \frac{1}{2}^{+} 0^{-}$interactions with similar results [8], the R-invariant vertex depending on $\mathrm{A}$ and a new arbitrary parameter $\mathrm{Z}$. Therefore, the $\mathrm{R}$ invariant Lagrangian involves, besides A, the so-called "off-shell" parameters X,Y,Z.

There exist controversy in the literature regarding the quantization of spin 3/2 interacting theory. Whereas some authors [8] claim that the constraints and the requirement of a consistent quantization pick up particular values for the "off-shell" parameters a carefull analysis [13] shows that negative norm states cannot be avoided. Alternative approaches [14], disregard the arbitrariness of the spin $1 / 2$ componets of $\psi_{\mu}$, take $\mathrm{X}, \mathrm{Y}, \mathrm{Z}$ as free parameters and use them to fit the experimental data. 


\section{HEAVY BARYON CHIRAL PERTURBATION THEORY (HBChPT).}

We have seen that Baryon Chiral Perturbation Theory necessarily involves interacting spin $3 / 2$ fields, and we have also summarized the problem faced by the interacting spin $3 / 2$ field theory. The next step is to analyze the new ingredients that the heavy fermion approach brings to the scene. According to the results of the previous section, to lowest order in the chiral expansion, the Lagrangian is

$$
\mathcal{L}=\mathcal{L}_{\text {free }}+\mathcal{L}_{\frac{3}{2} \frac{1}{2} 0}+\mathcal{L}_{\frac{3}{2} \frac{3}{2} 0}
$$

the free Lagrangian is given in (2) and the R-invariant interactions read

$$
\begin{aligned}
& \mathcal{L}_{\frac{3}{2} \frac{1}{2} 0}=i \mathcal{C} \bar{\psi}^{\mu} O_{\mu \nu}(A, Z) \psi \Delta^{\nu}+\text { h.c. } \\
& \mathcal{L}_{\frac{3}{2} \frac{3}{2} 0}=i \mathcal{H} \bar{\psi}^{\mu} O_{\mu \nu \alpha}(A, X, Y) \psi^{\nu} \Delta^{\alpha}+\text { h.c. }
\end{aligned}
$$

where $\mathcal{C}$ and $\mathcal{H}$ are coupling constants. $O_{\mu \nu \alpha}(A, X, Y)$ is given by:

$$
O_{\mu \nu \alpha}(A, X, Y)=R_{\mu \beta}(f(A, X)) g^{\beta}{ }_{\sigma} \gamma_{\alpha} \gamma_{5} R_{\nu}^{\sigma}(f(A, Y))
$$

while $O_{\mu \nu}(A, Z)$ can be written as:

$$
O_{\mu \nu}(A, Z)=R_{\mu \nu}(f(A, Z))
$$

with

$$
f(A, V)=\frac{1}{2}(1+4 V) A+V ; \quad V=X, Y, Z .
$$

Instead of working the field theory involving heavy baryons, we derive the Feynman rules for the effective theory by carrying out the $\frac{1}{m}$ expansion of the Feynman rules for $\mathrm{BChPT}$ and assume that the latter deffine the effective Heavy Baryon Chiral Perturbation Theory [15]. Rather than repeating the 
procedure worked out by $\mathrm{J}-\mathrm{M}$, just recall that the spin $3 / 2$ fields of definite velocity are defined by:

$$
\psi_{v}^{\mu}(x)=e^{-i m v \cdot x} \psi^{\mu}(x)
$$

where $\mathrm{m}$ stands for the nucleon mass in the chiral limit, i.e. the decuplet Lagrangian contains an explicit decuplet mass term proportional to $\Delta m=$ $M-m$.

In the heavy baryon limit $(m \rightarrow \infty)$, writing $P_{\mu}=m v_{\mu}+k_{\mu}$ and keeping leading terms in the $1 / \mathrm{m}$ expansion, the propagator (3) reduces to

$$
\begin{aligned}
i \Delta_{\mu \nu}(P, A)=\left\{-g_{\mu \nu}\right. & +\frac{1}{3} \gamma_{\mu} \gamma_{\nu}-\frac{1}{3}\left(\gamma_{\mu} v_{\nu}-v_{\mu} \gamma_{\nu}\right) \\
& \left.+\frac{2}{3} v_{\mu} v_{\nu}\right\} \frac{P_{+}}{(v \cdot k-\Delta m+i \epsilon)}+\mathcal{O}\left(\frac{1}{m} ; A\right) \\
& \equiv i \Delta_{\mu \nu}^{0}+\mathcal{O}\left(\frac{1}{m} ; A\right)
\end{aligned}
$$

where

$$
P_{+}=\frac{1+\not k}{2}
$$

It is straigthforward to check that

$$
\gamma^{\mu} \Delta_{\mu \nu}^{0}=\Delta_{\mu \nu}^{0} \gamma^{\nu}=0
$$

Notice that relations (11) are reminiscent of the constraints (1) holding for the free spin $3 / 2$ fields. The important observation is that to leading order in the $1 / \mathrm{m}$ expansion, the spin $3 / 2$ field behaves - as far as the constraints is concerned-like an on shell field; even if it is propagating. This implies that terms of the Lagragian containing the "off-shell" parameters $\mathrm{X}, \mathrm{Y}, \mathrm{Z}$, give no contribution to the $\mathrm{S}$ matrix elements. Indeed, for any calculation regarding the Lagrangian (10), the vertices $O_{\mu \nu}$ and $O_{\mu \nu \alpha}$ connect to an external on-shell heavy baryon or to a heavy baryon propagator $\Delta_{\mu \nu}^{0}$. 
The constraint for the free field (1), or relation (11) for the heavy propagator ensure in both cases the vanishing of the terms involving the "off-shell" parameters.

These results suggests that to leading order in the $1 / \mathrm{m}$ expansion, the constraints $\gamma^{\mu} \psi_{\mu}=0$ do not get modified by the interaction. This lead us naturally to the question of the constraints in the interacting theory.

The equation of motion for the spin $3 / 2$ field derived from (10) is:

$$
W_{\mu} \equiv \Lambda_{\mu \nu} \psi^{\nu}+i \mathcal{C} O_{\mu \nu}(A, X, Y) \psi \Delta^{\nu}+i \mathcal{H} O_{\mu \nu \alpha} \psi^{\nu} \Delta^{\alpha}=0
$$

where

$$
\Lambda_{\mu \nu}=P_{\alpha} \Gamma_{\mu \nu}^{\alpha}(A)-M B_{\mu \nu}(A)
$$

The subsidiary conditions are derived by performing the following operations:

$$
\begin{aligned}
{\left[\frac{1}{1+2 A}\left((1+A) \frac{P}{M}-1\right) \gamma^{\mu}-\frac{2}{M} P^{\mu}\right] W_{\mu} } & =0 \\
\frac{1+A}{1+2 A} \gamma^{\mu} W_{\mu}-2 \gamma^{0} W_{0} & =0
\end{aligned}
$$

These equations can be written as:

$$
\begin{aligned}
& 3(1+2 A) M \gamma^{\mu} \psi_{\mu}=i \mathcal{C}\left(\gamma^{\mu}-\frac{2}{M} P^{\mu}\right) O_{\mu \nu}^{\prime}(Z) \psi \Delta^{\nu} \\
- & i \mathcal{H}\left\{\gamma^{\mu}-\frac{2}{M} P^{\mu}\right\} O_{\mu \beta}^{\prime}(X) \tau_{\nu \alpha}^{\beta}(A, Y) \psi^{\nu} \Delta^{\alpha} \\
- & \frac{1}{2}\left(\frac{2}{3} \vec{P} \cdot \vec{\gamma}-M\right)\left(3(A+1) \gamma_{0} \psi^{0}-(3 A+1) \vec{\gamma} \cdot \vec{\psi}\right)=P_{i} \psi_{3 / 2}^{i} \\
- & \frac{i \mathcal{C}}{2}\left((1+2 Z) \gamma_{\nu}-2 \gamma^{0} g_{0 \nu}\right) \psi \Delta^{\nu}-\frac{i \mathcal{H}}{2}\left((1+2 X) \gamma_{\beta}-2 \gamma^{0} g_{0 \beta}\right) \\
& \tau_{\nu \alpha}^{\beta}(A, Y) \psi^{\nu} \Delta^{\alpha}
\end{aligned}
$$


where

$$
\begin{aligned}
O_{\mu \nu}^{\prime}(Z) & =g_{\mu \nu}-\frac{1}{2}(1+2 Z) \gamma_{\mu} \gamma_{\nu} \\
\tau_{\nu \alpha}^{\beta}(A, Y) & =g^{\beta}{ }_{\sigma} \gamma_{\alpha} \gamma_{5} R_{\nu}^{\sigma}(f(A, Y)) \\
\psi_{3 / 2}^{i} & =\left(\delta^{i \ell}+\frac{1}{3} \gamma^{i} \gamma^{\ell}\right) \psi_{\ell}
\end{aligned}
$$

Note that Eq. (14) implies

$$
\gamma^{\mu} \psi_{\mu}=0+\mathcal{O}\left(\frac{1}{m}, Z, X, Y\right)
$$

in agreement with our previous guess. Futhermore from Eq. (14) is easily seen that "off-shell" ambiguities appear in the next to leading order of the $1 / \mathrm{m}$ expansion, hence "off-shell" ambiguities free calculations can be carried on only to lowest order in the $1 / \mathrm{m}$ expansion of the Baryon Chiral Lagrangian. An implication of our argument is the need to keep track separately of the expansion parameter $\mathrm{m}$ (the baryon mass $\approx 1 \mathrm{GeV})$, and $\Lambda_{\chi}(\approx$ $1 \mathrm{GeV}$ ) even if they are of the same order of magnitude. $\Lambda_{\chi}$ is the parameter for the chiral expansion, on which there are no other limitation than usefullness, whereas consistency of the spin $3 / 2$ theory restricts the $1 / \mathrm{m}$ expansion to the lowest order.

Summarizing, we pointed out that Heavy Baryon Chiral Perturbation Theory faces the problems of the inconsistecies related to the interacting spin $\frac{3}{2}$ field theory $[7,8,12,13]$. We argued, by considering the $\frac{1}{m}$ expansion of the Feynman rules, that only the lowest order of the $1 / \mathrm{m}$ expansion is free of the so called "off-shell" ambiguities. Furthermore, we have shown that the constraints - which assure the elimination of the unphysical degrees of freedom contained in the Rarita-Schwinger spinor- is spoiled by the interactions by terms of order $\left(\frac{1}{m}\right)$, implying thus consistency of $H B C h P T$ only to lowest order of the expansion. We have also stressed the pertinence of dealing separately with the chiral and the $1 / \mathrm{m}$ expansion. 


\section{REFERENCES}

1.- For a recent account see J.F. Donohgue, E. Golowich \& B.R. Holstein, "Dynamics of the standard model" Cambridge Univ. Press (1992).

2.- E. Jenkins \& A. Manohar Phys. Lett. B255 (1991), 558.

3.- For a review see. B. Grinstein in "Proceedings of the Workshop on High Energy Phenomenology" Eds. M.A. Pérez y R. Huerta. World Scientific. (1992), 161.

4.- M.N. Butler et.al. Nucl. Phys. B399 (1993) 85. See also ref. 2

5.- J.W. Bos et.al. Phys. Rev. D51, (1995), 6308.

6.- W. Rarita \& J. Schwinger. Phys. Rev. 60, 61 (1941)

7.- C. Fronsdal Nuovo Cimento (suppl) IX, 416 (1958). See also J. Urias, Ph D. tesis, Universite Católique de Louvain (1976), unpublished.

8.- L.M. Nath, B. Etemadi \& J.D. Kimel, Phys. Rev. D3, 2153 (1971)

9.- S. Kamefuchi, L. O’Raifeartaigh \& A. Salam Nucl. Phys. 28, 529 (1961).

10.- M. El. Amiri, G. López \& J. Pestieau, Nucl. Phys. A 543 (1992) 673.

11.- R.D. Peccei, Phys. Rev. 1811902 (1969), Phys. Rev. 1761812 (1968)

12.- K. Jhonson \& E.C.G. Sudharsan, Ann. Phys. 13, 126, (1961). G. Velo \& D. Zwanziger, Phys. Rev. 186, 1337 (1969).

13.- C.R. Hagen, Phys. Rev. D4, 2204 (1971).

14.- Davidson et. al Phys. Rev. D43, (1991), 71 \& M.G. Olsson and E.T. Ossypowski, Nucl. Phys. B87, 339 (1975), Phy. Rev. D17, 174 (1978).

15.- M.B. Wise in "New Symmetries in Strong Interactions". Lectures presented at the Lake Louise Winter Institute (1991). 